\title{
Revista Colombiana de

\section{Capítulo 13. Utilidad del mapeo tridimensional de arritmias ventriculares en cardiopatía de origen no isquémico}

\author{
Chapter 13. Usefulness of three-dimensional mapping \\ in ventricular arrhythmias in non-ischemic cardiomyopathy
}

\author{
Francisco Villegas ${ }^{\mathrm{a}, \mathrm{b}, \mathrm{c}, *}$ y Carlos Gómez ${ }^{\mathrm{d}}$ \\ a Cardiología-Electrofisiología, Hospital Universitario Departamental Santa Sofía de Caldas, Manizales, Colombia \\ ${ }^{b}$ Fundación Cardiovascular de Colombia-Instituto del Corazón de Manizales, Manizales, Colombia \\ c SES-Hospital de Caldas, Manizales, Colombia \\ d Clínica Medellín, Medellín, Colombia
}

Recibido el 17 de noviembre de 2015; aceptado el 18 de enero de 2016

\section{Introducción}

La cardiopatía no isquémica podría definirse como aquella que se presenta en ausencia de enfermedad coronaria significativa (estenosis mayor del 75\%) o de infarto de miocardio previo. No podría considerarse a la cardiopatía no isquémica como una enfermedad única, sino como el resultado final de múltiples entidades como: enfermedad valvular cardiaca, cardiopatía hipertensiva, sarcoidosis, amiloidosis, enfermedad de Chagas, cardiopatía alcohólica, enfermedades infecciosas, miocarditis, cardiopatía arritmogénica del ventrículo derecho, cardiopatía periparto, cardiopatía viral y cardiopatía dilatada idiopática propiamente dicha, que es aquella en la que no se han podido documentar ninguna de las causas anteriores. Además, podrían incluirse en esta categoría las fases finales de la cardiopatía hipertrófica. En el $50 \%$ de los sujetos afectados, es posible encontrar una causa etiológica que explique la cardiopatía; el $50 \%$ restante se consideran casos idiopáticos. Independiente del origen, el corazón sufre un incremento en la masa miocárdica y una pérdida del grosor de la pared ventricular ${ }^{1}$.

Al igual que en la cardiopatía de origen isquémico, la no isquémica se encuentra claramente relacionada con un incremento en el riesgo de desarrollar arritmias ventriculares, incluidas taquicardia y fibrilación ventricular. Pero en contraste con la cardiopatía isquémica, el sustrato electrofisiológico que permite la instauración y el mantenimiento de estas arritmias no está claramente definido y aunque la taquicardia por reentrada rama a rama se ha considerado típica de esta patología, cerca del $80 \%$ de los casos parecen originarse de alteraciones miocárdicas y están relacionadas más con la presencia de cicatrices que permiten el establecimiento de circuitos de reentrada 2 .

En estos pacientes con cardiopatía no isquémica, la fibrosis miocárdica, el desarreglo en la disposición de las miofibrillas y las anormalidades en la función de la membrana de los miocitos son factores importantes para el desarrollo del sustrato que permite el establecimiento de estas arritmias. 


\section{Arritmogénesis}

La taquicardia ventricular sostenida se asocia con la existencia de fibrosis miocárdica extensa y con la presencia de áreas de anisotropía no uniformes que comprenden tanto el endocardio como el epicardio y al igual que en aquellos sujetos con cardiopatía isquémica los circuitos están relacionados con áreas con electrogramas de bajo voltaje compatibles con tejido cicatricial ${ }^{3}$. Los estudios de mapeo con catéter en sujetos con cardiopatía no isquémica, muestran circuitos de reentrada que se establecen alrededor de áreas de cicatrices profundas en el miocardio o a nivel epicárdico, con una frecuencia mucho mayor que en aquellos pacientes con cardiopatía isquémica en quienes el compromiso suele ser más de tipo transmural o con mayor compromiso en el endocardio. Estos corazones tienen áreas de fibrosis que no son excitables eléctricamente y crean regiones de bloqueo en la conducción que al alternarse con áreas de miocardio viable pueden promover el establecimiento de circuitos de reentrada. Estas áreas son identificables con el uso de resonancia magnética nuclear con realce tardío y se han correlacionado con las áreas donde se demuestra que está ubicado el circuito de la taquicardia 4 .

La causa de la fibrosis en cardiopatía no isquémica no está claramente establecida y suelen encontrarse regiones parcheadas con reemplazo de tejido normal por tejido fibrótico, sin grandes áreas de confluencia de tejido cicatricial como ocurre en la cardiopatía isquémica. También se han demostrado áreas de bajo voltaje sugestivas de tejido cicatricial en pacientes con cardiopatía no isquémica y presencia de taquicardia por reentrada rama a rama, pero el área de cicatriz parece ser más pequeña.

Comparadas con las áreas encontradas en cardiopatía isquémica, las de cicatriz en cardiopatía no isquémica tienden a ser más pequeñas y menos confluentes; el número total de segmentos con compromiso transmural es menor y tienen menos compromiso endocárdico ${ }^{2}$. En los sujetos isquémicos se establece un frente de progresión en la necrosis que va del subendocardio al epicardio (con áreas de cicatriz mayores en el endocardio que en el epicardio que usualmente están confinadas a un territorio vascular). En contraste, las cicatrices en cardiopatía no isquémica tienden a mostrar predilección por el miocardio propiamente dicho o por el epicardio. En lugar de cicatrices densas con haces de miocardio sobreviviente, las cicatrices en cardiopatía no isquémica son parcheadas y tienen menos canales protegidos ${ }^{5}$.

A pesar de lo expuesto, existen varias similitudes entre los sujetos con cardiopatía no isquémica y aquellos con historia de infarto de miocardio. La presencia de áreas de bajo voltaje se observa en todos los casos al igual que la presencia de canales o istmos protegidos, lo que permite realizar el mapeo de los circuitos que permiten el establecimiento de la taquicardia.

La incidencia de cardiopatía no isquémica varía de 5 a 8 por 100.000 personas/año y su mortalidad a 5 años se estima en un $20 \%$. La muerte súbita de origen cardiaco es la responsable de aproximadamente el $30 \%$ de estos decesos y aunque la taquicardia y la fibrilación ventricular se consideran los mecanismos más frecuentes de muerte súbita cardiaca en este tipo de cardiopatía, la bradicardia, los trastornos de la conducción aurículo-ventricular, la embolia pulmonar y la actividad eléctrica sin pulso, entre otras causas, dan cuenta del
$50 \%$ de las muertes consideradas como súbitas en pacientes con falla cardiaca avanzada 6 .

\section{Cardiodesfibrilador implantable}

El uso del cardiodesfibrilador implantable se ha convertido en la terapia de elección con el fin de disminuir el riesgo de muerte súbita arrítmica en estos pacientes; sin embargo, su uso no previene la aparición de episodios arrítmicos, solo conlleva a su terminación por medio de estimulación antitaquicardia o de terapia con descarga eléctrica, la cual es dolorosa y se ha demostrado que no solamente disminuye la calidad de vida de los sujetos, sino que puede estar relacionada en forma independiente con una disminución en la supervivencia de los mismos, como consecuencia del daño que las descargas repetidas infligen en un corazón, por demás ya comprometido. Aunque el beneficio de la terapia con desfibrilador no es tan evidente como en aquellos pacientes con cardiopatía isquémica, en aquellos que tienen cardiopatía no isquémica y episodios demostrados de taquicardia ventricular sostenida o de fibrilación ventricular sin una causa secundaria corregible o en aquellos con disfunción ventricular severa con fracción de eyección menor al $35 \%$ a pesar de la terapia farmacológica óptima, el uso del desfibrilador está claramente recomendado en la actualidad ${ }^{7}$.

\section{Estudio electrofisiológico}

Teniendo en cuenta la incapacidad de los cardiodesfibriladores para evitar la aparición de los fenómenos arrítmicos, al igual que la de la terapia antiarrítmica, la ablación con catéter ha ido posicionándose como una estrategia útil para el manejo de las arritmias ventriculares en estos pacientes. La indicación más frecuente para la ablación de taquicardia ventricular en pacientes con cardiopatía no isquémica son las descargas frecuentes (incluida la tormenta eléctrica) a pesar del tratamiento antiarrítmico. Algunos pueden debutar con episodios de taquicardia ventricular sostenida antes de la implantación de un desfibrilador y en ellos también estaría indicada la realización de ablación con catéter; no obstante, al tener en cuenta el riesgo de arritmias recurrentes debido al carácter progresivo de las enfermedades que llevan a cardiopatía no isquémica, se considera que la ablación no es un substituto para el implante del desfibrilador y que siempre deberá considerarse su implantación en estos pacientes incluso si el resultado inicial de la ablación fue satisfactorio.

Una vez se ha decidido la realización de la intervención por medio de ablación en estos pacientes, durante el estudio electrofisiológico inicial siempre deberá descartarse taquicardia ventricular por reentrada rama a rama, así se tenga evidencia de morfologías de taquicardia que impliquen un circuito de reentrada. Ya realizada la exclusión o el tratamiento de la taquicardia por reentrada rama a rama, el proceso de mapeo y ablación de la o las taquicardias ventriculares (múltiples morfologías) en estos sujetos, se lleva a cabo con los mismos principios generales que se usan para el manejo de las arritmias ventriculares en cardiopatía isquémica, teniendo en cuenta que la probabilidad de encontrar circuitos de reentrada de ubicación intramiocárdica o epicárdica es muchísimo mayor y que por tanto el personal que vaya a 
realizar la intervención esté en capacidad de abordar estrategias de ablación que permitan lidiar con este tipo de suceso en el sustrato arritmogénico.

La obtención de un electrocardiograma durante la taquicardia se considera supremamente valioso ya que permite tener una guía de la posible localización anatómica del sitio de salida de la taquicardia y además tratar de establecer con anterioridad la necesidad de abordaje epicárdico, el cual, como se comentó, es muy frecuente en estos pacientes. De no ser posible y si el paciente ya tiene un cardiodesfibrilador implantado, el análisis de la morfología y la longitud de ciclo de la taquicardia clínica permitirá tratar de elucidar durante el procedimiento cuál de las posibles múltiples morfologías que suelen inducirse en estos pacientes (promedio de $3 \pm 1$ morfologías) es la que está generando los episodios espontáneos (clínicos).

\section{Utilidad del mapeo tridimensional}

\section{Mapeo endocárdico tridimensional}

El uso de los sistemas de mapeo tridimensional en estos pacientes se considera fundamental a tal punto que no se concibe dentro del ejercicio de la electrofisiología moderna intentar realizar ablación de arritmias ventriculares en pacientes con cardiopatía no isquémica con el uso de sistemas de mapeo convencionales (es decir, guiados solamente por señales eléctricas y guía fluoroscópica únicamente $)^{8}$. Además del mapeo tridimensional, la posibilidad de contar con sistemas de guía en tiempo real como la ecocardiografía intracardiaca, da ventajas adicionales a la hora del mapeo y la ablación, y permite una aproximación más segura a este tipo de pacientes de por sí muy complejos.

Los sistemas de mapeo tridimensional permiten, en aquellos casos con arritmias ventriculares toleradas desde un punto de vista hemodinámico, realizar mapeo de activación con la visualización de mapas isocronales que ayudan a estable- cer no solo el sitio de primoactivación, sino a vislumbrar el recorrido del frente de onda eléctrico a medida que despolariza la cavidad ventricular (por medio de dicho mapa de colores) y por ende facilitar el entendimiento de los posibles circuitos de reentrada en dicha cavidad. Ayuda, así mismo, en la verificación de un circuito de macrorreentrada como causa de la arritmia versus un origen focal, ya que la presencia del encuentro de la actividad más precoz adyacente con la actividad más tardía (siempre y cuando las variables que determinan el mapeo estén configuradas de forma adecuada) permite comprobar este fenómeno?.

Los sistemas de mapeo tridimensional facilitan además la visualización de mapas de activación, que dejan ver el desplazamiento del frente de onda ya no como un mapa de colores, sino como el movimiento en el espacio de la despolarización ventricular, dando así un entendimiento aún más completo del circuito de reentrada.

Toda esta información, sumada a las maniobras de encarrilamiento de la taquicardia, con la ventaja de poder marcar los sitios donde se hace la estimulación durante dichas maniobras, al igual que poder dejar marcados en la cavidad ventricular los sitios donde se realizan maniobras de topoestimulación (pace mapping), permite caracterizar de manera muy detallada los circuitos involucrados en la génesis de la arritmia y hace posible señalar los sitios donde se realiza ablación ${ }^{9,10}$ (fig. 1).

En aquellos pacientes con arritmias ventriculares no toleradas desde el punto de vista hemodinámico, en quienes las maniobras de mapeo no pueden ser realizadas durante la taquicardia, los sistemas de mapeo tridimensional son imprescindibles ya que son la forma en que se puede desarrollar el denominado "mapeo de sustrato". En este tipo de estrategia, el mapeo se efectúa durante el ritmo de base del paciente (bien sea sinusal, estimulado o con información obtenida durante ambos) y no durante la taquicardia como tal. El mapeo de sustrato hace referencia a la tipificación del sustrato de la taquicardia ventricular basado en la identificación de electrogramas locales anormales tales como electrogramas
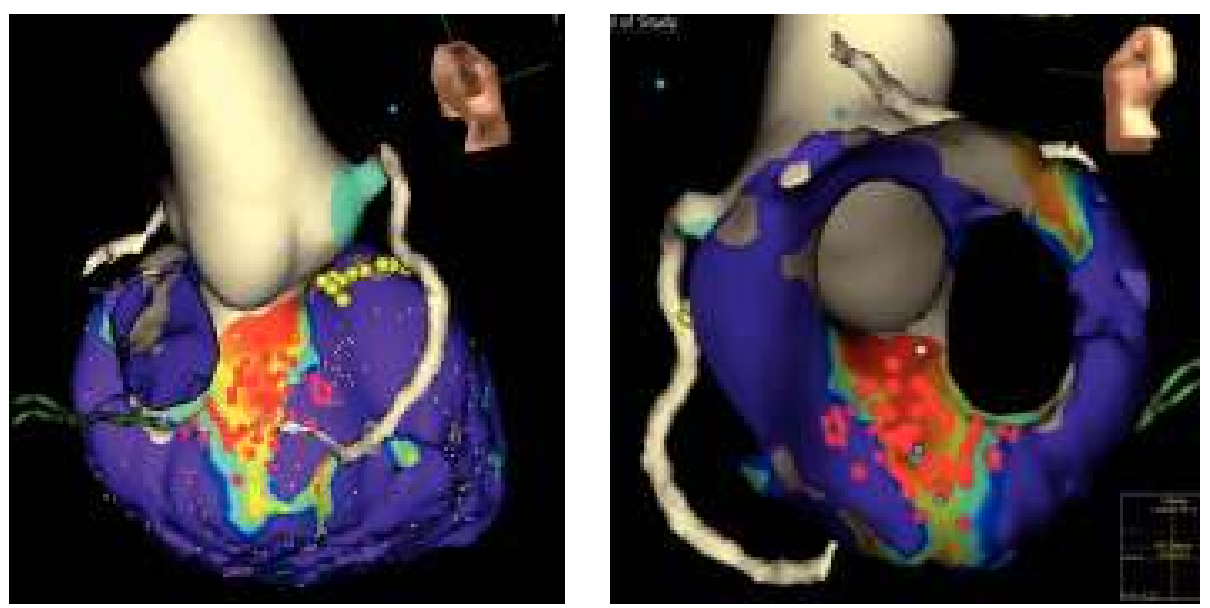

Figura 1 Mapa endocárdico y epicárdico con sistema EnSite integrado con reconstrucción 3-D por tomografía axial computarizada del ventrículo izquierdo en un paciente con miocardiopatía dilatada idiopática que pone en evidencia una cicatriz septal discreta. Se aprecia el origen de las arterias coronarias; el sistema de conducción se referencia con los puntos amarillos. El corte virtual endoscópico permite apreciar los anillos valvulares y la continuidad mitro-aórtica. Los puntos rojos representan las aplicaciones de radiofrecuencia de la homogenización de la cicatriz. 
fraccionados, electrogramas con múltiples potenciales y electrogramas con componentes aislados, así como a la de electrogramas locales de baja amplitud durante el ritmo sinusal (mapeo de voltaje) y de canales que hacen parte del circuito de reentrada que da origen a la taquicardia ${ }^{11-13}$ (fig. 2).

Los potenciales aislados durante el ritmo sinusal son un reflejo del tejido cicatricial subyacente. Aunque grandes áreas de cicatrización no son frecuentes en los pacientes con cardiopatía no isquémica al contrario de la isquémica, la presencia de fibrosis intersticial extensa es común cuando se analiza desde el punto de vista histológico. Porciones de tejido fibroso podrían servir como barreras eléctricas y arrojar electrogramas fragmentados. Los potenciales aislados puede ser identificados en muchos pacientes (no en todos) con cardiopatía no isquémica, y son más frecuentes en aquellos con miocardiopatía arritmogénica del ventrículo derecho y en casos con sarcoidosis, mas no así en pacientes con cardiopatía dilatada idiopática. A pesar de estas limitaciones se ha encontrado que aquellos pacientes en quienes se identifican estos potenciales y se tienen en cuenta como sitios de ablación, tienen mejor resultado a corto y mediano plazo luego de la ablación que aquellos en quienes la caracterización del sustrato arrítmico no incluye los potenciales ${ }^{13,14}$.

Además de su uso en pacientes inestables, el mapeo de sustrato brinda información valiosa durante el mapeo de activación y las maniobras de encarrilamiento en pacientes con arritmias bien toleradas desde el punto de vista hemodinámico, ya que permite enfocarse en los sitios más probables de origen de la arritmia y por tanto ayuda a minimizar el tiempo que el paciente estará en taquicardia ventricular.

\section{Mapeo epicárdico tridimensional}

El mapeo tridimensional también se ha mostrado como una herramienta sumamente valiosa en el mapeo epicárdico de estas arritmias. Este permite caracterizar el sustrato de la arritmia a nivel epicárdico al igual que lo hace en el endocardio. En estos pacientes la necesidad de realizar mapeo y ablación es muchísimo mayor que en los sujetos con cardiopatía isquémica. La decisión de efectuar abordaje epicárdico podría variar de acuerdo con varias situaciones. En centros con poca experiencia en mapeo y ablación epicárdica, es frecuente realizar un abordaje endocárdico para el mapeo y la ablación de la arritmia de modo que en caso de recurrencia

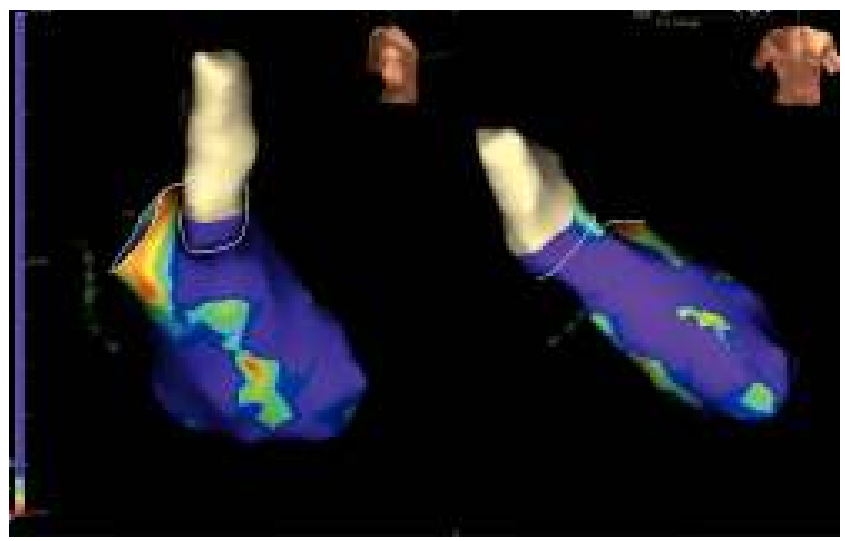

Figura 2 Mapa de voltaje donde se aprecian zonas normales (color magenta) y zonas de cicatriz. posprocedimiento se podría planear en un segundo tiempo el abordaje epicárdico. Sin embargo y teniendo en cuenta que la necesidad de mapeo y ablación epicárdica en este tipo de pacientes es frecuente, es común que en centros con amplia experiencia en mapeo epicárdico este abordaje se realice desde el principio (fig. 1).

Una alternativa para tratar de establecer la necesidad de un abordaje epicárdico desde el principio sería la presencia de signos electrocardiográficos durante la taquicardia ventricular (tales como una seudoonda delta igual o mayor a 34 milisegundos, una deflexión intrinsecoide en V2 igual o mayor de 85 milisegundos, una duración del RS igual o mayor a 121 $\mathrm{ms}$, una onda $\mathrm{Q}$ en la derivación $\mathrm{DI}$ o un índice de deflexión máxima igual o mayor a 0,55$)^{10}$. Sin embargo, la no disponibilidad de un electrocardiograma durante el episodio de taquicardia podría imposibilitar esta aproximación.

La utilización de resonancia magnética nuclear con realce tardío que indique la presencia de compromiso epicárdico sería de mucha utilidad al planear la necesidad de un abordaje epicárdico ${ }^{15,16}$. No obstante, en estos pacientes un cardiodesfibrilador implantado podría dificultar la realización de este método diagnóstico, aunque existe evidencia de que en una gran proporción de pacientes este examen puede llevarse a cabo siguiendo ciertos protocolos en forma rigurosa ${ }^{17}$.

Durante el procedimiento, el mapeo tridimensional también puede sugerir la necesidad de un abordaje epicárdico. La realización de un mapa de voltaje unipolar a nivel endocavitario puede ayudar a predecir la existencia de cicatrices epicárdicas y por ende la necesidad de un abordaje a este nivel ${ }^{18,19}$.

Con base en lo expuesto queda claro que el uso de sistemas de mapeo tridimensional no solo es útil, sino necesario, al momento de realizar procedimientos de mapeo y ablación en pacientes con arritmias ventriculares en el contexto de cardiopatía de origen no isquémico.

\section{Recomendaciones}

\section{Clase I}

- Se recomienda el uso de sistemas de mapeo tridimensional en pacientes que serán llevados a ablación de taquicardias ventriculares en presencia de miocardiopatía dilatada no isquémica (nivel de evidencia $C$ ).

\section{Bibliografía}

1. Hamilton RM, Azevedo ER. Sudden cardiac death in dilated cardiomyopathies. Pacing Clin Electrophysiol. 2009;32 Suppl 2:S32-40.

2. Aliot EM, Stevenson WG, Mendral-Garrote JM, et al. EHRA/HRS Expert Consensus on Catheter Ablation of Ventricular Arrhythmias: developed in a partnership with the European Heart Rhythm Association (EHRA), a Registered Branch of the European Society of Cardiology (ESC), and the Heart Rhythm Society (HRS); in collaboration with the American College of Cardiology (ACC) and the American Heart Association (AHA). Heart Rhythm. 2009;6:886-933.

3. Yokokawa M, Tada H, Koyama K, et al. The characteristics and distribution of the scar tissue predict ventricular tachycardia 
in patients with advanced heart failure. Pacing Clin Electrophysiol. 2009;32:314-22

4. Yokokawa M, Tada H, Koyama K, et al. Nontransmural scar detected by magnetic resonance imaging and origin of ventricular tachycardia in structural heart disease. Pacing Clin Electrophysiol. 2009;32 Suppl 1:S52-6.

5. Nakahara S, Tung R, Ramirez RJ, et al. Characterization of the arrhythmogenic substrate in ischemic and nonischemic cardiomyopathy implications for catheter ablation of hemodynamically unstable ventricular tachycardia. J Am Coll Cardiol. 2010;55:2355-65.

6. Vest RN, Gold MR. Risk stratification of ventricular arrhythmias in patients with systolic heart failure. Curr Opin Cardiol. 2010;25:68-75.

7. Myerburg RJ, Reddy V, Castellanos A. Indications for implantable cardioverter-defibrillators based on evidence and judgment. J Am Coll Cardiol. 2009;54:747-63.

8. Tanaka Y, Huang SKS, Gerstenfeld EP. Advanced catheter three dimensional mapping systems. En: S.K.S Huang, JM Miller. Catheter Ablation of Cardiac Arrhythmias. 3rd ed. Philadelphia: Elsevier Saunders; 2014. p. 68-86.

9. Tanimoto K, Hsia HH. How to map and ablate hemodynamically tolerated ventricular tachycardias. En: Al Ahmad A, Callans D, Hsia HH, Natale A, Oserof O, Wang PJ, editors. Hands-On Ablation: The Experts' Approach. Mineapolis: Cardiotext; 2013.

10. Bogun FM, Crawford TC. Ablation of ventricular tachycardia associated with nonischemic cardiomyopathy. En: Huang SK, Miller JM. Catheter Ablation of Cardiac Arrhythmias. 3rd ed. Philadelphia: Elsevier Saunders; 2014. p. 289-311.

11. Tzou WS, Marchlinski FE. How to map and ablate unstable ventricular tachycardia: The University of Pennsylvania Approach. En: Al Ahmad A, Callans D, Hsia HH, Natale A, Oserof O, Wang PJ, editors. Hands-On Ablation: The Experts' Approach. Mineapolis: Cardiotext; 2013. p. 323-56.
12. Hsia HH, Tanimoto K. How to utilize electroanatomical mapping to identify critical channels for ventricular tachycardia ablation. En: Al Ahmad A, Callans D, Hsia HH, Natale A, Oserof O, Wang PJ, editors. Hands-On Ablation: The Experts 'Approach. Mineapolis: Cardiotext; 2013. p. 288-301.

13. Castellanos E, Almendral J, De Diego C. How to map and ablate ventricular tachycardia using delayed potential in sinus rhythm. En: Al Ahmad A, Callans D, Hsia HH, Natale A, Oserof O, Wang PJ, editors. Hands-On Ablation: The Experts Approach. Mineapolis: Cardiotext; 2013. p. 302-22.

14. Kuhne M, Abrams G, Sarrazin JF, et al. Isolated potentials and pace-mapping as guides for ablation of ventricular tachycardia in various types of nonischemic cardiomyopathy. J Cardiovasc Electrophysiol. 2010;21:1017-23.

15. Bogun FM, Desjardins B, Good E, et al. Delayed enhanced magnetic resonance imaging in nonischemic cardiomyopathy: utility for identifying the vetricular arrhythmia substrate. J Am Coll Cardiol. 2009;53:1138-45.

16. Piers SR, Tao Q, Van Huls Van Taxis CF, Schalij MJ, Van der Geest RJ, Zeppenfeld K. Contrast-enhanced MRI derived scar patterns and associated ventricular tachycardias in nonischemic cardiomyopathy: implications for the ablation strategy. Cir Arrhytm Electrophysiol. 2013;6:875-83.

17. Nazarian $S$, Hansford R, Roguin $A$, et al. A prospective evaluation of a protocol for magnetic resonance imaging of patients with implanted cardiac devices. Ann Inter Med. 2011;155:415-4.

18. Hutchinson MD, Gerstenfeld EP, Desjardins B, et al. Endocardial unipolar voltaje mapping to detect epicardial VT substrate in patients with nonischemic cardiomyopathy. Cir Arrhythm Electrophysiol. 2011;4:49-55.

19. Polin GM, Haqqani H, Tzou W, et al. Endocardial unipolar voltaje mapping to identify epicardial substrate in arrhythmogenic right ventricular displasia/cardiomyopathy. Heart Rhythm. 2010;8:76-83. 